\title{
Obesidad severa en adolescentes peruanos: análisis de la Encuesta Nacional de Hogares (ENAHO), 2009-2010
}

\author{
Severe obesity in peruvian adolescents: analysis of the National \\ Household Survey (ENAHO), 2009- 2010
}

\author{
Jaime Pajuelo Ramírez ${ }^{1, a}$, Harold L. Torres Aparcana ${ }^{2, b}$, Fernando Bravo Rebatta ${ }^{3,4, c}$, Rosa Agüero Zamora, \\ ${ }^{1}$ Investigador independiente \\ ${ }^{2}$ Clínica San Felipe. Lima, Perú. \\ ${ }^{3}$ Centro Nacional de Alimentación y Nutrición, Instituto Nacional de Salud. Lima, Perú. \\ ${ }^{4}$ Universidad Católica Sedes Sapientiae. Lima, Perú. \\ a Médico cirujano, ORCID: https://orcid.org/0000-0002-9312-2087 \\ ${ }^{\mathrm{b}}$ Médico cirujano, ORCID: https://orcid.org/0000-0002-7489-4145 \\ ${ }^{\circ}$ Nutricionista \\ ${ }^{d}$ Médico cirujano, ORCID: https://orcid.org/0000-0002-7969-1208
}

An Fac med. 2019;80(4):470-4 / DOI: https://doi.org/10.15381/anales.v80i4.17253

\section{Correspondencia: \\ Jaime Pajuelo Ramirez \\ japara18@yahoo.com}

Recibido: 15 de julio 2019

Aceptado: 6 de noviembre 2019

Publicación en línea: 28 de diciembre

2019

Conflictos de interés: Los autores declaran no tener conflictos de interés.

Fuente de financiamiento: Autofinanciado

Citar como: Pajuelo J, Torres H, Bravo $F$, Agüero R. Obesidad severa en adolescentes peruanos: análisis de la Encuesta Nacional de Hogares (ENDES) 2009-2010. An Fac med. 2019;80(4):470-4. DOI: https://doi. org/10.15381/anales.v80i4.17253

\section{Resumen}

Introducción. La obesidad infantil es un problema de salud pública en nuestro país; sin embargo, la situación de la población entre 10 a 19 años ha sido poco estudiada. Objetivo. Determinar la prevalencia de obesidad y obesidad severa en adolescentes peruanos. Métodos. Estudio de datos secundarios provenientes de la Encuesta Nacional de Hogares 2009-2010. Se utilizó el índice de masa corporal (IMC) definiendo obesidad con un valor de IMC $\geq$ percentil 95 (p95). Obesidad severa (OS) mediante dos criterios: IMC $\geq$ percentil 99 (p99) y el $120 \%$ del valor correspondiente al percentil 95 del IMC. Resultados. El 7,6\% presentó obesidad, el 2,4 y $1,4 \%$ presentaron OS según los criterios citados. Las mayores prevalencias de OS se evidenciaron en varones ( $3 \%$ vs $1,8 \%$ ), en el grupo de 10 a 14 años ( $3,4 \%$ vs $2 \%$ ), en Lima Metropolitana ( $4,2 \%$ vs $2,4 \%$ ), área urbana ( $3,2 \%$ vs $1,9 \%)$, no pobres ( $3,1 \%$ vs $1,9 \%$ ) y en ciudades de menos de $1000 \mathrm{msnm}(3,5 \%$ vs $2.1 \%)$. Se encontró mayor razón de prevalencia (RP) de OS en los varones y no pobres. Tener de 15 a 19 años, residir en sierra o selva, área rural y ser pobre se asoció independientemente a menor RP. Conclusión. La prevalencia de OS fue baja a predominio de varones, en el grupo de 10 a 14 años, en la costa y Lima Metropolitana, en áreas urbanas, en los considerados no pobres y en los que viven por debajo de los $1000 \mathrm{msnm}$.

Palabras claves: Obesidad Pediátrica; Obesidad Mórbida; Encuestas Nutricionales; Medicina del Adolescente (fuente: DeC BIREME).

\section{Abstract}

Introduction. Childhood obesity is a Public Health problem in our country; instead of that, the situation of population aged between 10-19 years-old has been poorly studied. Objective. To determine the prevalence of obesity and severe obesity in Peruvian adolescents. Methods. A secondary study of data from the National Household Survey 2009-2010. The Body Mass Index (BMI) was used to define obesity as a BMI value $\geq 95$ percentile. Severe obesity (SO) was defined using two criteria, BMI $\geq 99$ p and $120 \%$ of the value corresponding to $95 \mathrm{p}$ of BMI. Results. 7,6\% presented obesity, and 2,4 and 1,4\% were diagnosed with SO according to the mentioned criteria. The highest prevalence of SO was evidenced in men ( $3 \%$ vs. 1,8\%), age group of 10 to 14 years-old (3,4\% vs. $2 \%$ ), people who are living in Lima Metropolitana ( $4,2 \%$ vs. $2,4 \%$ ), or in a urban area $(3,2 \%$ vs. $1,9 \%)$, non-poor ( $3,1 \%$ vs. $1,9 \%)$ and in cities less than 1000 meters above sea level (3,5\% vs. 2,1\%). A higher prevalence ratio (PR) of SO was found in men and non-poor. Having an age between 15 to 19 years old, residing in the mountains or jungle, rural area and being poor, were independently associated with a lower PR. Conclusion. The prevalence of SO was low, with a predominance of men, in the 10 to 14 year old group, on the coast and Metropolitan Lima, in urban areas, in those considered non-poor and in those living below 1000 meters above sea level.

Keywords: Pediatric Obesity; Obesity, Morbid; Nutrition Surveys; Adolescent Medicine (source: MeSH NLM). 


\section{INTRODUCCIÓN}

En las últimas décadas, la obesidad ha sido considerada como un problema de salud pública no solamente en los países desarrollados sino también en aquellos donde sus economías se encuentran en transición ${ }^{(1)}$. El primer estudio realizado en el Perú que proporcionó información sobre el estado nutricional de la población fue la Evaluación del Estado Nutricional del Poblador Peruano en 1975. Esta información mostró que la prevalencia de obesidad fue de $4,4 \%$ en los niños de 6 a 9 años, de 1,3\% en los adolescentes y del $9 \%$ en los adultos. Estudios posteriores han mostrado que estas prevalencias se han incrementado y en lo que se refiere a los adolescentes, la obesidad ha pasado a un $7,5 \%{ }^{(2)}$.

Existen evidencias que las personas con obesidad presentan mayores riesgos que los que tienen sobrepeso o peso normal. Estas complicaciones cuando aparecen a temprana edad y no se toman las medidas correspondientes, comprometerían muy seriamente su salud futura ${ }^{(3)}$. Asímismo, la obesidad infantil afecta prácticamente a todos los sistemas de órganos en corto y largo plazo, incluso aquellas complicaciones que se pensaban solo se presentaban en la adultez ${ }^{(4)}$.

Sin embargo, el uso de una sola categoría para identificar a estos pacientes no toma en cuenta que en su interior pueden existir variaciones en cuanto a la severidad. En ese sentido existen reportes que muestran que, por ejemplo, las personas con obesidad mórbida o severa, presentan factores de riesgo metabólico como el C-HDL, los triglicéridos, hemoglobina glicosilada y presión arterial significativamente mayores a quienes tienen sobrepeso y obesidad ${ }^{(5,6,7)}$. De la misma manera, representan un importante impulsor de la diabetes tipo 2 que se desarrolla en adultos jóvenes en una población india americana ${ }^{(8)}$.

Asimismo, resultados de diversos estudios en niños y adolescentes evidencian que la obesidad severa (OS) comparada con peso normal, sobrepeso y obesidad, se asocia con altos niveles de estrés oxidativo e inflamación como son el C-LDL oxidado, la proteína $C$ reactiva y la IL-6, resistencia a la insulina, y con ello mayor riesgo cardiovascular en este grupo ${ }^{(9)}$. En forma similar, los niveles de marcadores de daño oxidativo como la manolinaldehido y los grupos carbonilo plasmático fueron más altos en los que presentaban mayor obesidad ${ }^{(10)}$.

Por otro lado, se ha demostrado en los que tienen OS la existencia de un compromiso en la función microvascular que podría atribuirse a un deterioro de su capacidad vasoconstrictora, constituyendo un signo temprano de disfunción endotelial ${ }^{(11)}$. Lamentablemente no hay un consenso referente a su identificación y esto responde principalmente a dos razones: la primera es la utilización de distintos criterios diagnósticos en referencia al valor percentilar del punto de corte ${ }^{(12-16)}$ y la segunda es que al usar diferentes poblaciones de referencia los valores que corresponden a los percentiles difieren.

El objetivo del presente estudio fue determinar la prevalencia de obesidad y obesidad severa en adolescentes en Perú, utilizando datos de la Encuesta Nacional de Hogares (ENAHO) llevada a cabo entre los años 2009 a 2010.

\section{MÉTODOS}

\section{Diseño del estudio}

Se realizó un estudio observacional, transversal y analítico. La información del presente estudio se basa en los datos proporcionados por la Encuesta Nacional de Hogares (ENAHO 2009-2010) llevada a cabo por el Instituto Nacional de Estadística e Informática (INEI) conjuntamente con el Centro Nacional de Alimentación y Nutrición (CENAN) del Instituto Nacional de Salud que dependen del Ministerio de Salud del Perú (17).

\section{Población y muestra}

La población alcanzó a 14886 adolescentes de las cuales se excluyó a 83 gestantes quedando 14803, comprendidos entre los 10 a 19 años. Esta encuesta realizó un muestreo probabilístico, estratificado y multietápico. Para el cálculo del tamaño de la muestra se evalúo la precisión estadística obtenida en la muestra ENAHO 2009-2010 para estimar algunas características de la vivienda y del hogar, características de los miembros del hogar, educación, salud, empleo, ingreso y gastos del hogar; nivel de confianza al $95 \%$ y precisión $5 \%$. También se consideró el efecto de diseño y las tasas de no respuestas para realizar los ajustes respectivos

\section{Variables de estudio}

Dentro de las variables antropométricas se consideró al peso y talla, los cuales fueron medidos con la metodología internacionalmente aceptada. Con ambas medidas se calculó el índice de masa corporal (IMC) expresado en $\mathrm{kg} / \mathrm{m}^{2}$. Los valores del IMC fueron contrastados con la población de referencia de la Organización Mundial de la Salud (OMS) ${ }^{(18)}$ y se definió como obesidad a las medidas de IMC $\geq$ percentil 95. De los múltiples criterios para definir obesidad severa (OS), se eligieron a dos de ellos por cuanto representan a los más utilizados en la literatura: uno es el IMC $\geq$ percentil $99^{(12)}$ y el otro, el $120 \%$ del valor correspondiente al percentil 95 del IMC ${ }^{(13)}$.

Otras variables incluidas en nuestro estudio fueron: género, edad (de 10 a 14 y de 15 a 19 años), región natural (costa, sierra, selva y Lima Metropolitana), área de residencia (urbana y rural), pobreza (pobre y no pobre) y altitud (0 a 999, 1000 a 2999 y 3000 msnm y más). La pobreza se midió por un enfoque monetario. Se consideró hogar pobre cuando el gasto per cápita era inferior a la línea de pobreza (hogares que cubren sus necesidades en alimentación, pero no cubren sus necesidades en otros bienes y servicios como educación, salud, vestido, calzado, entre otros), se incluyó en este grupo a los considerados como pobres extremos ${ }^{(19)}$.

\section{Análisis estadístico}

Se empleó el software Stata Statistical Software: Release 12 (STATACorp Lp, College Station, TX, $2011^{\circledR}$ ) para los métodos estadísticos utilizados en este estudio. Para el análisis se tuvo en consideración el efecto de diseño para muestras complejas, empleándose el comando (svy). Se estimó prevalencias y sus res- 
pectivos intervalos de confianza. El análisis bivariado y multivariado se realizó mediante modelos lineales generalizados para determinar la razón de prevalencia (RP) de manera directa. En el análisis multivariado se realizó ajustes por género, grupo etario, región, nivel socioeconómico, área de residencia y altitud. Para todos estos análisis se consideró un nivel de confianza del $5 \%$.

\section{RESULTADOS}

La población estudiada fue de 14803 adolescentes comprendidos entre los 10 y 19 años. La mayor proporción correspondió al género masculino $(52,1 \%)$, al grupo etario de 10 a 14 años (50,1\%), población costera 49,4\% (costa y Lima Metropolitana), residentes en áreas urbanas $(68,8 \%)$, los considerados no pobres $(62,6 \%)$ y a los que viven en ciudades ubicadas por debajo de los $1000 \mathrm{msnm}$ (60\%) (Tabla 1).

Tabla 1. Distribución de los registros incluidos en el estudio, de acuerdo a variables sociodemográficas, de la Encuesta Nacional de Hogares (ENAHO) 2009-2010

\begin{tabular}{|c|c|c|c|}
\hline Características & & $\mathrm{n}$ & $\%$ \\
\hline \multicolumn{4}{|l|}{ Género } \\
\hline & Masculino & 7467 & 52,1 \\
\hline & Femenino & 7336 & 47,9 \\
\hline \multicolumn{4}{|l|}{ Edad (años) } \\
\hline & $10-14$ & 8109 & 50,1 \\
\hline & $15-19$ & 6694 & 49,9 \\
\hline \multicolumn{4}{|l|}{ Región natural } \\
\hline & Costa & 3494 & 22,9 \\
\hline & Sierra & 6474 & 37,2 \\
\hline & Selva & 3606 & 13,5 \\
\hline & $\begin{array}{c}\text { Lima } \\
\text { Metropolitana }\end{array}$ & 1229 & 26,5 \\
\hline \multicolumn{4}{|c|}{ Área de residencia } \\
\hline & Urbana & 7911 & 68,8 \\
\hline & Rural & 6892 & 31,2 \\
\hline \multicolumn{4}{|l|}{ Pobreza } \\
\hline & Pobres & 6507 & 37,5 \\
\hline & No pobres & 8296 & 62,6 \\
\hline \multicolumn{4}{|l|}{ Altitud (msnm) } \\
\hline & $0-999$ & 7670 & 60,0 \\
\hline & 1000- 2999 & 3345 & 17,5 \\
\hline & 3000 y más & 3788 & 22,6 \\
\hline
\end{tabular}

msnm: metros sobre el nivel del mar.

(*) Se consideró el efecto de diseño, es por ello que los porcentajes pueden no coincidir con los $n$.
A nivel nacional, la prevalencia de obesidad (IMC $\geq$ p 95) alcanzó el 7,6\% (IC $95 \% 6,9-8,3)$, además fue similar en ambos géneros. Se encontró mayores prevalencias en el grupo de 10 a 14 años, a nivel de la costa y Lima Metropolitana, en residentes del área urbana, en no pobres y a menos de 1000 msnm (Tabla 2).

Respecto a la OS, se encontró mayor prevalencia con el criterio IMC $\geq$ p 99 que con un IMC $\geq$ de $120 \%$ del p 95 (2,4\% vs $1,4 \%)$. A diferencia de la obesidad en general, la obesidad severa tuvo mayor prevalencia en varones, llegando incluso a duplicar la prevalencia en mujeres. El resto de factores estudiados tuvo las mismas tendencias que la obesidad en general (Tabla 2).

Respecto a OS, En el análisis multivariado los adolescentes varones presentaron $75 \%$ mayor prevalencia que las mujeres; asimismo, los no pobres tuvieron una prevalencia $80 \%$ mayor que los po-

Tabla 2. Prevalencia (IC al 95\%) de obesidad y de obesidad severa en adolescentes, de acuerdo a diferentes criterios, y según variables socio-demográficas. Encuesta Nacional de Hogares (ENAHO) 2009-2010.

\begin{tabular}{|c|c|c|c|c|c|c|c|}
\hline \multirow[t]{2}{*}{ Características } & & \multicolumn{2}{|c|}{$\begin{array}{l}\text { Obesidad } \\
\text { (IMC>p95) }\end{array}$} & \multicolumn{2}{|c|}{$\begin{array}{c}\text { Obesidad severa } \\
\text { (IMC } \geq \text { p99) }\end{array}$} & \multicolumn{2}{|c|}{$\begin{array}{c}\text { Obesidad severa } \\
\text { (IMC } \geq 120 \% \text { del } \\
\text { p95) }\end{array}$} \\
\hline & & $\%$ & IC $95 \%$ & $\%$ & IC $95 \%$ & $\%$ & IC $95 \%$ \\
\hline \multicolumn{8}{|l|}{ Género } \\
\hline & Masculino & 7,6 & $6,7-8,7$ & 3,0 & $2,4-3,6$ & 1,8 & $1,4-2,4$ \\
\hline & Femenino & 7,5 & $6,6-8,5$ & 1,7 & $1,3-2,2$ & 0,9 & $0,7-1,2$ \\
\hline \multicolumn{8}{|l|}{ Edad (años) } \\
\hline & $10-14$ & 9,5 & $8,5-10,6$ & 3,4 & $2,8-4,0$ & 2,0 & $1,6-2-6$ \\
\hline & $15-19$ & 5,6 & $4,9-6,5$ & 1,3 & $1,0-1,8$ & 0,8 & $0,5-1,2$ \\
\hline \multicolumn{8}{|l|}{ Región natural } \\
\hline & Costa & 10,8 & $9,4-12,2$ & 3,7 & $2,9-4,7$ & 2,5 & $1,8-3,4$ \\
\hline & Sierra & 3,7 & $3,1-4,3$ & 0,7 & $0,5-1,0$ & 0,3 & $0,2-0,6$ \\
\hline & Selva & 4,4 & $3,6-5,3$ & 1,1 & $0,7-1,5$ & 0,6 & $0,4-0,9$ \\
\hline & $\begin{array}{l}\text { Lima } \\
\text { Metropolitana }\end{array}$ & 11,8 & $9,9-14,0$ & 4,2 & $3,2-5,5$ & 2,4 & $1,6-3,4$ \\
\hline \multicolumn{8}{|c|}{ Área de residencia } \\
\hline & Urbana & 9,9 & $9,0-10,9$ & 3,2 & $2,7-3,8$ & 1,9 & $1,5-2,4$ \\
\hline & Rural & 2,4 & $2,0-2,9$ & 0,5 & $0,3-0,7$ & 0,3 & $0,2-0-4$ \\
\hline \multicolumn{8}{|l|}{ Pobreza } \\
\hline & $\begin{array}{l}\text { Pobreza } \\
\text { extrema }\end{array}$ & 4,1 & $3,4-5,0$ & 1,1 & $0,7-1,6$ & 0,5 & $0,3-0,9$ \\
\hline & No pobres & 9,6 & $8,7-10,7$ & 3,1 & $2,6-3,7$ & 1,9 & $1,5-2,4$ \\
\hline \multicolumn{8}{|l|}{ Altitud (msnm) } \\
\hline & 0- 999 & 10,0 & $9,0-11,2$ & 3,5 & $2,9-4,1$ & 2,1 & $1,6-2,6$ \\
\hline & 1000- 2999 & 5,1 & $4,2-6,2$ & 1,0 & $0,7-1,5$ & 0,5 & $0,3-0,8$ \\
\hline & 3000 y más & 2,9 & $2,3-3,6$ & 0,5 & $0,3-0,8$ & 0,3 & $0,1-0,6$ \\
\hline Nacional & & 7,6 & $6,9-8,3$ & 2,4 & $2,0-2,8$ & 1,4 & $1,1-1,7$ \\
\hline
\end{tabular}

bres. Por otro lado, quienes presentaron una menor prevalencia de OS fueron: el grupo de 15 a 19 años con un 64\% menos que los de 10 a 14 años; los habitantes de la costa y selva con un $59 \%$ menos respecto a los habitantes de la costa; y los residentes en zona rural $68 \%$ menos que los de zona urbana. Todas estas asociaciones fueron independientes y significativas. En el análisis bivariado, residir en zonas de mayor altitud se asoció a menor riesgo de OS, pero esta asociación no se mantuvo al ajustar por los otros factores sociodemográficos (Tabla 3).

\section{DISCUSIÓN}

La obesidad es un problema de salud pública que trae consigo una disminución en la calidad de vida y, además un incremento en los costos para la sociedad y los sistemas de salud en su conjunto ${ }^{(20)}$. 
Tabla 3. Modelo bivariado y multivariado de la asociación entre obesidad severa en adolescentes y factores sociodemográficos. Encuesta Nacional de Hogares (ENAHO) 2009-2010.

\begin{tabular}{|c|c|c|c|c|c|}
\hline Características & & RPc & IC 95\% & $\mathbf{R P a}$ & IC 95\% \\
\hline \multicolumn{6}{|l|}{ Género } \\
\hline & Masculino & 1.76 & $1.28-2.42$ & 1.75 & $1.28-2.39$ \\
\hline & Femenino & 1 & Ref. & 1 & Ref. \\
\hline \multicolumn{6}{|l|}{ Edad (años) } \\
\hline & 10- 14 & 1 & Ref. & 1 & Ref. \\
\hline & 15- 19 & 0,40 & $0,28-0,57$ & 0,36 & $0,25-0,51$ \\
\hline \multicolumn{6}{|l|}{ Región natural } \\
\hline & Costa & 1 & Ref. & 1 & Ref. \\
\hline & Sierra & 0,18 & $0,12-0,27$ & 0,41 & $0,19-0,88$ \\
\hline & Selva & 0,29 & $0,19-0,45$ & 0,41 & $0,26-0,63$ \\
\hline & Lima metropolitana & 1,13 & $0,79-1,62$ & 0.94 & $0,65-1,35$ \\
\hline \multicolumn{6}{|l|}{ Área de residencia } \\
\hline & Urbana & 1 & Ref. & 1 & Ref. \\
\hline & Rural & 0,14 & $0.09-0,22$ & 0,32 & $0,20-0,54$ \\
\hline \multicolumn{6}{|l|}{ Pobreza } \\
\hline & Pobre & 1 & Ref. & 1 & Ref. \\
\hline & No pobres & 2,93 & $1,92-4,49$ & 1,80 & $1,14-2,83$ \\
\hline \multicolumn{6}{|l|}{ Altitud (msnm) } \\
\hline & $0-999$ & 1 & Ref. & 1 & Ref. \\
\hline & 1000- 2999 & 0,30 & $0,20-0,46$ & 1,02 & $0,52-1,98$ \\
\hline & 3000 y más & 0,13 & $0,07-0,24$ & 0,47 & $0,19-1,18$ \\
\hline
\end{tabular}

RPc: Razón de prevalencia cruda; Rpa: Razón de prevalencia ajustada; Ref.: Referencia

En los adolescentes, la obesidad se ha incrementado pasando de $1,3 \%$ en el año $1975^{(2)}$ al 7,6\% reportado en el presente estudio. Si bien no se tiene información anterior respecto a la presencia de OS, podemos deducir que esta podría haberse aumentado por cuanto también se ha incrementado la prevalencia de obesidad. La literatura reporta que, en algunos países como Australia, China, Reino Unido, Francia, Países Bajos, Nueva Zelanda, Suecia, Suiza y Estados Unidos (USA) la prevalencia de obesidad viene mostrando una meseta ${ }^{(21)}$; y pese a ello, en Nueva Zelanda ${ }^{(22)}$ y USA ${ }^{(23)}$ la prevalencia de OS se ha incrementado.

En nuestro estudio elegimos como uno de los criterios para identificar la OS, el recomendado por un comité de expertos de EE.UU. que propuso como definición a aquellos que tuviesen un IMC $\geq$ percentil 99, lo que identificó un subgrupo de obesos con un factor de riesgo cardiovascular adverso mostrando que el seguimiento de la adiposidad en la edad adulta fue extremadamente fuerte en estos individuos ${ }^{(12)}$. El otro criterio fue el sugerido por la American Heart Association de un IMC $\geq 120 \%$ del percentil $95^{(13)}$.
Optando por el criterio de un IMC $\geq$ p99, la prevalencia encontrada fue relativamente baja (2,4\%), representando un $30 \%$ de los obesos reportados (7,6\%). Otros estudios mostraron prevalencias más altas como el National Health and Nutrition Examination Surveys (NHANES 1999-2004) de 3,8\% (24), The Health Study con $6,9 \%{ }^{(25)}$; mientras que otras investigaciones muestran resultados casi iguales, como en Italia, dentro de la iniciativa de la Vigilancia de la Obesidad Infantil de la Oficina Regional de la OMS para Europa, iniciada en 2007 con 2,2\% ${ }^{(26)}$ y en Nueva Zelanda con un $2,9 \%$, aunque en este último país usaron como criterio un IMC $\geq$ percentil 99,7 ${ }^{(22)}$. Utilizando el punto de corte, de un IMC $\geq 120 \%$ del p95, la OS alcanzó el 1,4\%; prevalencia menor en relación a otros reportes como el Korea $\mathrm{Na}$ tional Health and Nutrition Examination Surveys (K-NHANES 2007-2014) con un $6,8 \%{ }^{(6)}$, en el NHANES $1999-2012$ un $8 \%$ (23), NHANES del 2014 8,7\% (27), NHANES pero del ciclo $2015-20167,9 \%{ }^{(28)}$ y en Australia 2,0\% ${ }^{(29)}$.

En nuestro estudio los varones presentaron mayores prevalencias de OS (IMC $\geq$ p99) que las mujeres (3 vs $1,8 \%$ ). Ten- dencia parecida también se observó en el NHANES 1999-2004 (4,6 vs 2,9\%) ${ }^{(24)}$, en Nueva Zelanda $(3,9 \text { vs } 2,6 \%)^{(22)}$, y en adolescentes de 10 a 11 años (3,9 vs 2,9\%) (14), así como en niños de 2 a 19 años para el NHANES 2015-2016 $\left(9,7\right.$ vs 7,0) ${ }^{(28)}$, en China (2,7 vs $1,3 \%)^{(30)}$ y en Europa, en el Childhood Obesity Surveillance Initiative (COSI), en niños de 6 a 9 años, utilizando otros criterios diagnósticos, encontraron lo mismo ${ }^{(31)}$. Esta mayor prevalencia implica de algún modo un incremento en el riesgo, lo que se confirma con la razón de prevalencia ajustada por las otras variables, obtenida en esta investigación (RP ajustada 1,75 IC 95\% 1,28-2,39).

Respecto a la relación de la OS con la pobreza, nuestros resultados indican que existió 3 veces más riesgo de ser OS en los considerados no pobres (RPcruda 2,93 IC $95 \% 1,92-4,49)$ y que al ajustarlo con las otras variables disminuye muy poco en relación a lo mencionado, existiendo en cada una de ellas significancia estadística; es decir, que los menos afectados son los considerados pobres, lo que representa una situación parecida a la encontrada en el estudio de Zhang et al en China ${ }^{(30)}$. Sin embargo, en otras investigaciones se ha evidenciado todo lo contrario, teniendo las personas en situación de pobreza, prevalencias de obesidad más altas ${ }^{(32)}$. Adicionalmente, también se ha reportado que las personas con altos ingresos y educación superior son lo que menos OS presentan, encontrándose este diagnóstico más asociado a los niveles socio económicos bajos ${ }^{(33)}$ caracterizado como el "entorno obesogénico", puede ser más generalizado en los estratos sociales en los EE.UU.

En contraposición, presentaron menor riesgo de tener OS los que vivían en áreas rurales y en ciudades por encima de los 1000 msnm; esto respondería a que en esos escenarios se presentan en menor proporción los factores que determinan a la obesidad, hecho ya reportado en las encuestas nacionales ${ }^{(2)}$. Cuando se ajusta con las otras variables (RPa), las áreas rurales, siguen mostrando una asociación significativa caso contrario sucede con la variable altitud. En lo que corresponde a las regiones naturales, solamente los que vivían en sierra y selva se encontraban protegidos, lo mismo que el grupo de 15 a 19 años. 
Los problemas que conlleva la obesidad no solamente deben pensarse en términos de morbimortalidad sino también en el estigma que a menudo la acompaña; así, Spahlholz mostró que las percepciones de discriminación que sufren por su exceso de peso son bastante comunes y sus consecuencias negativas son altamente relevantes dentro de la sociedad ${ }^{(34)}$. La situación se agrava por el hecho de que hasta un $79 \%$ no reconoce que su hijo se encuentra excedido de peso, y cuando lo reconocen el $41 \%$ no aprecia el riesgo que representa para la salud ${ }^{(35)}$.

Si bien las prevalencias encontradas son bajas, la tendencia que se viene observando es al incremento como respuesta a la falta de control de los factores que la generan. Si bien no existe una proyección de la OS en el grupo de adolescentes, sí existe para el grupo de adultos. Finkelstein reportó que para el año 2030, en relación a lo conocido del 2010, la prevalencia de obesidad mórbida (IMC $40 \mathrm{~kg} / \mathrm{m}^{2}$ ) pasará de 4,7 a $8,8 \%{ }^{(36)}$.

Se debe considerar como limitación de nuestro estudio la falta de información de años previos, con lo cual no es posible determinar la tendencia de la OS. Asimismo, el presentar datos de una encuesta nacional del año 2010, no refleja el actual estado de la obesidad severa en adolescentes peruanos.

Concluimos que la presencia de OS fue baja y su mayor localización fue en el género masculino, en el grupo de 10 a 14 años, en la costa y Lima Metropolitana, en áreas urbanas, en los considerados no pobres y en los que viven por debajo de los 1000 msnm. La altitud fue la única variable que no mostró asociación significativa estadística.

\section{REFERENCIAS BIBLIOGRÁFICAS}

1. GBD 2015 Obesity Collaborators, Afshin A, Forouzanfar MH, Reitsma MB, Sur P, Estep K, et al. Health Effects of Overweight and Obesity in 195 Countries over 25 Years. N Engl J Med. 2017;377(1):13-27. DOI: 10.1056/ NEJMoa1614362

2. Pajuelo-Ramírez J. La obesidad en el Perú. Anales de la Facultad de Medicina. 2017;78(2):179-85. DOI: 10.15381/anales.v78i2.13214

3. Pajuelo R. J, Arbañil H. H, Sánchez G. J, Gamarra G. D, Torres A. L, Pando A. R, et al. Riesgo cardiovascular en población infantil con sobrepeso y obesidad. Anales de la Facultad de Medicina. 2013;74(3):181-6. DOI: https:// doi.org/10.15381/anales.v74i3.2632
4. Daniels SR. Complications of obesity in children and adolescents. Int J Obes (Lond). 2009;33 Suppl 1:S6065. DOI: $10.1038 /$ ijo.2009.20

5. Skinner AC, Perrin EM, Moss LA, Skelton JA. Cardiometabolic Risks and Severity of Obesity in Children and Young Adults. N Engl J Med. 2015;373(14):1307-17. DOI: 10.1056/NEJMoa1502821

6. Cho WK, Han K, Ahn MB, Park Y-M, Jung MH, Suh B-K, et al. Metabolic risk factors in Korean adolescents with severe obesity: Results from the Korea National Health and Nutrition Examination Surveys (K-NHANES) 20072014. Diabetes Res Clin Pract. 2018;138:169-76. DOI: 10.1016/.diabres.2018.01.030

7. Lo JC, Chandra M, Sinaiko A, Daniels SR, Prineas RJ, Maring B, et al. Severe obesity in children: prevalence, persistence and relation to hypertension. Int J Pediatr Endocrinol. 2014;2014(1):3. DOI: 10.1186/1687-98562014-3

8. Tanamas SK, Reddy SP, Chambers MA, Clark EJ, Dunnigan DL, Hanson RL, et al. Effect of severe obesity in childhood and adolescence on risk of type 2 diabetes in youth and early adulthood in an American Indian population. Pediatr Diabetes. 2018;19(4):622-9. DOI: 10.1111/pedi.12627

9. Norris AL, Steinberger J, Steffen LM, Metzig AM, Schwarzenberg SJ, Kelly AS. Circulating oxidized LDL and inflammation in extreme pediatric obesity. Obesity (Silver Spring). 2011;19(7):1415-9. DOI: 10.1038/ oby.2011.21

10. Codoñer-Franch $P$, Boix-Garcia L, Simó-Jordá R, Del Castillo-Villaescusa C, Maset-Maldonado J, VallsBellés V. Is obesity associated with oxidative stress in children? Int J Pediatr Obes. 2010;5(1):56-63. DOI: 10.3109/17477160903055945

11. Schlager O, Willfort-Ehringer A, Hammer A, Steiner S, Fritsch M, Giurgea A, et al. Microvascular function is impaired in children with morbid obesity. Vasc Med. 2011;16(2):97-102. DOI: 10.1177/1358863X11400780

12. Barlow SE, Expert Committee. Expert committee recommendations regarding the prevention, assessment, and treatment of child and adolescent overweight and obesity: summary report. Pediatrics. 2007;120 Suppl 4:S164-192. DOI: 10.1542/peds.2007-2329C

13. Flegal KM, Wei R, Ogden CL, Freedman DS, Johnson $\mathrm{CL}$, Curtin LR. Characterizing extreme values of body mass index-for-age by using the 2000 Centers for Disease Control and Prevention growth charts. Am J Clin Nutr. 2009;90(5):1314-20. DOI: 10.3945/ajcn.2009.28335

14. Ells LJ, Hancock C, Copley VR, Mead E, Dinsdale H, Kinra S, et al. Prevalence of severe childhood obesity in England: 2006-2013. Arch Dis Child. 2015;100(7):631-6. DOI: 10.1136/archdischild-2014-307036

15. Cole TJ, Lobstein T. Extended international (IOTF) body mass index cut-offs for thinness, overweight and obesity. Pediatr Obes. 2012;7(4):284-94. DOI: 10.1111/.2047-6310.2012.00064.x

16. El Mouzan MI, Foster PJ, Al Herbish AS, Al Salloum AA, Al Omer AA, Qurachi MM, et al. Prevalence of overweight and obesity in Saudi children and adolescents. Ann Saudi Med. 2010;30(3):203-8. DOI: 10.4103/0256-4947.62833

17. Instituto Nacional de Estadística e Informática (INEI). Encuesta Nacional de Hogares (ENAHO 2009-2010). Lima: INEI; 2010.

18. de Onis M, Onyango AW, Borghi E, Siyam A, Nishida C, Siekmann J. Development of a WHO growth reference for school-aged children and adolescents. Bull World Health Organ. 2007;85(9):660-7. DOI: 10.2471/ blt.07.043497

19. Instituto Nacional de Estadistica e Informática (INEI). Perú-Encuesta Nacional de Hogares sobre Condiciones de Vida y Pobreza (2017). Lima: INEI; 2017.
20. Kelly AS, Barlow SE, Rao G, Inge TH, Hayman LL, Steinberger J, et al. Severe obesity in children and adolescents: identification, associated health risks, and treatment approaches: a scientific statement from the American Heart Association. Circulation. 2013;128(15):1689-712. DOI: 10.1161/ CIR.0b013e3182a5cfb3

21. Olds T, Maher C, Zumin S, Péneau S, Lioret S, Castetbon $\mathrm{K}$, et al. Evidence that the prevalence of childhood overweight is plateauing: data from nine countries. Int J Pediatr Obes. 2011;6(5-6):342-60. DOI: 10.3109/17477166.2011.605895

22. Shackleton N, Milne BJ, Audas R, Derraik JGB, Zhu T, Taylo $\mathrm{RW}$, et al. Improving rates of overweight, obesity and extreme obesity in New Zealand 4-year-old children in 2010-2016. Pediatr Obes. 2018;13(12):766-77. DOI: 10.1111/jpo.12260

23. Skinner AC, Skelton JA. Prevalence and trends in obesity and severe obesity among children in the United States, 1999-2012. JAMA Pediatr. 2014;168(6):561-6. DOI: 10.1001/ jamapediatrics.2014.21

24. Skelton JA, Cook SR, Auinger P, Klein JD, Barlow SE. Prevalence and trends of severe obesity among US children and adolescents. Acad Pediatr. 2009;9(5):322-9. DOI: 10.1016/j. acap.2009.04.005

25. Marcus MD, Baranowski T, DeBar LL, Edelstein S, Kaufman FR, Schneider M, et al. Severe obesity and selected risk factors in a sixth grade multiracial cohort: the HEALTHY study. J Adolesc Health. 2010;47(6):604-7. DOI: 10.1016/j. jadohealth.2010.04.017

26. Lombardo FL, Spinelli A, Lazzeri G, Lamberti A, Mazzarella G, Nardone P, et al. Severe obesity prevalence in 8- to 9-year-old Italian children: a large population-based study. European Journal of Clinical Nutrition. 2015;69(5):603-8. DOI: 10.1038/ ejcn.2014.188

27. Skinner AC, Perrin EM, Skelton JA. Prevalence of obesity and severe obesity in US children, 1999-2014. Obesity (Silver Spring). 2016;24(5):1116-23. DOI: 10.1002/oby.21497

28. SkinnerAC, RavanbakhtSN, Skelton JA, Perin EM, Armstrong SC Prevalence of Obesity and Severe Obesity in US Children, 19992016. Pediatrics. 2018;141(3). DOI: 10.1542/peds.2017-3459

29. Garnett SP, Baur LA, Jones AMD, Hardy LL. Trends in the Prevalence of Morbid and Severe Obesity in Australian Children Aged 7-15 Years, 1985-2012. PLOS ONE. 2016;11(5):e0154879. DOI: 10.1371/journal.pone.0154879

30. Zhang Y-X, Chu Z-H, Li S-Y, Zhao J-S, Zhou J-Y. Trends in the Prevalence of Morbid Obesity among Children and Adolescents in Shandong, China, 1995-2014. J Trop Pediatr. 2018;64(1):60-6. DOI: 10.1093/tropej/fm×030

31. Spinelli A, Buoncristiano M, Kovacs VA, Yngve A, Spirosk I, Obreja G, et al. Prevalence of Severe Obesity among Primary School Children in 21 European Countries. OFA. 2019;12(2):244-58. DOI: 10.1159/000500436

32. De Spiegelaere M, Hennart $P$, Dramaix M. Social class and obesity in 12-year-old children in Brussels: Influence of gender and ethnic origin. Eur J Pediatr. 1998;157(5):432-5. DOI: 10.1007/s004310050845

33. Booth HP, Charlton J, Gulliford MC. Socioeconomic inequality in morbid obesity with body mass index more than $40 \mathrm{~kg} /$ m2 in the United States and England. SSM Popul Health. 2017:3:172-8. DOI: 10.1016/.ssmph.2016.12.012

34. Spahlholz J, Baer N, König H-H, Riedel-Heller SG, LuckSikorski C. Obesity and discrimination - a systematic review and meta-analysis of observational studies. Obes Rev. 2016;17(1):43-55. DOI: 10.1111/obr.12343

35. Park MH, Falconer CL, Saxena S, Kessel AS, Croker H, Skow $A$, et al. Perceptions of health risk among parents of overweight children: a cross-sectional study within a cohort. Prev Med. 2013;57(1):55-9. DOI: 10.1016/j.ypmed.2013.04.002

36. Finkelstein EA, Khavjou OA, Thompson H, Trogdon JG, Pan L, Sherry B, et al. Obesity and severe obesity forecasts through 2030. Am J Prev Med. 2012;42(6):563-70. DOI: 10.1016/j. amepre.2011.10.026 